\title{
Assessment of Airway Remodeling in Asthma: Volumetric Versus Surface Quantification Approaches
}

\author{
Amaury Saragaglia, Catalin Fetita, and Françoise Prêteux \\ ARTEMIS Project Unit, INT, Groupe des Ecoles des Télécommunications \\ 9 rue Charles Fourier, 91011 Evry Cedex, France
}

\begin{abstract}
This paper develops a volumetric quantification approach of the airway wall in multi-detector computed tomography (MDCT), exploiting a 3D segmentation methodology based on patient-specific deformable mesh model. A comparative study carried out with respect to a reference $2 \mathrm{D} / 3 \mathrm{D}$ surface quantification technique underlines the clinical interest of the proposed approach in assessing airway remodeling in asthmatics and in evaluating the efficiency of therapeutic protocols.
\end{abstract}

\section{Introduction}

Automated quantification of airway responses to pharmacologic agents is the key issue in establishing and evaluating therapeutic protocols in severe and mild asthma or chronic obstructive pulmonary disease (COPD). The inflammation of the airways is known to induce airway remodeling, such indicator being commonly used in clinical practice to confirm the diagnosis of asthma. The current challenge for $\mathrm{CT}$ in asthma is to assess airway reactivity and wall remodeling in response to an excitation agent (e.g., bronchoprovocation, bronchodilatation, response to changes in lung volumes), that is, to accurately quantify bronchial lumen and wall variations. Efficient imaging methods are required to provide accurate and reproducible measurements of the inner and outer edges of the airway walls. This can be challenging for the small caliber, thin wall airways, and even for normal size bronchi because of inherent partial volume effect in the image acquisition.

Semi-automated or automated methods have been developed for airway wall segmentation. They apply to section images of bronchi and can be roughly classified into three categories : full-width at half-maximum (FWHM) 1], patternbased [2 3|4] and shape-independent approaches. FWHM-based methods estimate the inner/outer contour of the wall by investigating the grey-level profile of rays cast from the bronchus center. However, it has been shown in [5] that FWHM can yield inaccurate measurements for small airways or for those having very thin walls (10\% to $15 \%$ of lumen diameter). Pattern-based approaches were used to automatically detect circular or ellipsoidal shape patterns. These methods are fast and seem to be efficient on phantom studies and excised animal lungs in case of circular and elliptical shapes detection, but do not apply accurately to general clinical data. 
More recent methods exploits morphological operators to achieve shape-independent, accurate quantification for irregular wall bronchi. In [6], such a technique is applied in a $2 \mathrm{D} / 3 \mathrm{D}$ framework, to $2 \mathrm{D}$ image sections orthogonal to the bronchus axis. Here, the medial axis of bronchi is computed from a 3D segmentation of the airway lumen. In this way, the quantification is independent of CT acquisition plane and can be theoretically obtained at any desired spatial location, which is essential for investigating diffuse diseases such asthma.

Note however that, even performed in a cross-sectional plane, bronchial lumen/wall surface quantification methods may suffer from several drawbacks related either to the uncertainty of medial axis estimation accuracy or to CT acquisition parameters discussed further on in this paper. 3D quantification makes it possible to overcome such limitations, by investigating the bronchus wall volume variation caused by airway remodeling.

This paper develops a volumetric quantification approach of the airway wall in MDCT, relying on the 3D segmentation of the inner/outer bronchus wall surface using patient-specific deformable mesh models. Such a method is then evaluated with respect to phantom image and real CT data, and compared with the $2 \mathrm{D} / 3 \mathrm{D}$ technique developed in 6]. The paper is organized as follows. Section 2 summarizes the clinical investigation framework and the reference 2D/3D method, while Section 3 is dedicated to a detailed description of the developed volumetric approach. A comparative discussion of volumetric versus surface quantification accuracy is developed and illustrated in Section 4 for phantom and clinical data. Concluding remarks are stated in Section 5 .

\section{Clinical Framework}

\subsection{Data Acquisition and Preprocessing}

Low-dose MDCT acquisitions were performed in severe and mild asthmatics in the framework of a therapeutic follow-up study conducted in a large hospital group in Paris. Images obtained at $65 \%$ of the total lung capacity with $0.6 \mathrm{~mm}$ collimation and $0.3 \mathrm{~mm}$ reconstruction interval focused on the right lung in a 18-20 cm field of view, resulting in quasi-isotropic volume data, free from cardiac motion artifacts [9]. The objective was to evaluate bronchial wall remodeling on (sub-)subsegmental bronchi at several locations spread throughout the lung.

\subsection{Reference 2D/3D Quantification Method}

In order to evaluate the performance and clinical interest of a volumetric quantification approach, a surface quantification method was selected as reference from the recent literature 6], for comparison purposes. Achieving an accurate estimation of bronchial parameters (lumen and wall areas), requires to perform the surface quantification analysis in a cross-section plane orthogonal to the bronchus axis. A preprocessing step first performs the 3D airway lumen segmentation from the MDCT data using multi-resolution morphological filtering and energy-based modeling [7. Then, a full description of this arborescent structure is achieved by 
computing the medial axis [8] (Fig. 1(a)). Medial axis provides the navigation and interaction tools required for selecting bronchial segments and generating cross-section images at appropriate spatial resolution and sampling intervals along bronchus axis (Fig. 1(b) . Only axial images outside of the subdivision zones are selected for analysis. The quantification of the bronchial parameters is performed independently on each cross-section image. It exploits a segmentation method combining mathematical morphology operators and energy-based contour matching [6]. The quantification results are then averaged for each bronchus. Fig. 1(c) shows bronchial inner/outer wall segmentation result.

As reported in 910, when cross-tested on synthetic and natural database, the selected 2D segmentation approach overperformed the FWHM and the patternbased methods in terms of quantification accuracy and robustness with respect to the presence of homologous artery. Therefore such a method is chosen as the reference one for evaluating the performance of the new developed volumetric segmentation and quantification approach.

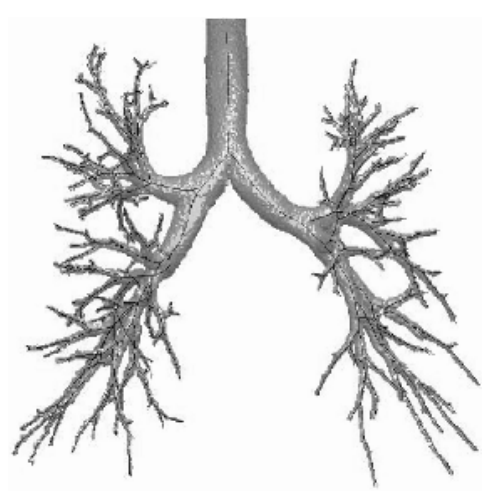

(a)

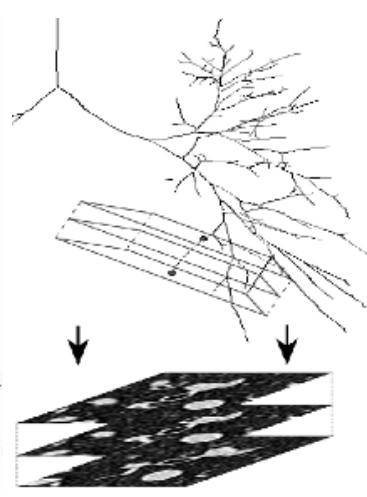

(b)
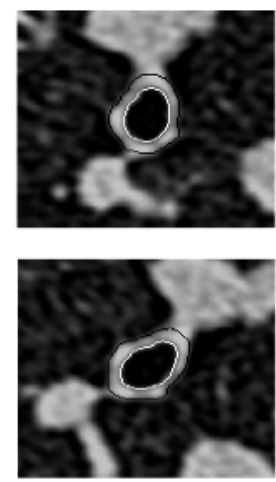

(c)

Fig. 1. Airway tree and 2D/3D quantification of bronchial parameters: (a) 3D Segmentation and corresponding medial axis, (b) Cross section image reconstruction orthogonally to the medial axis at the selected sampling locations, (c) 2D wall segmentation on sample data

\section{The Developed Volumetric Approach}

The volumetric quantification approach developed in this paper relies on the 3D segmentation of the bronchial wall using a patient-specific deformable mesh model. Starting from the 3D segmentation of the airway lumen obtained in $\S$ 2.2, a mesh model of the inner airway wall is built-up. In order to preserve the topology and geometry of the small bronchi involved in the quantification process and to obtain an regular mesh, a restricted Delaunay triangulation approach is applied [11,12] with a specific adaptative distance criterion [13].

The outer surface of the bronchial wall is then segmented by deforming the inner wall mesh model (Fig. 2(a) conditional to image data and shape regularity 
constraints. A motion equation is assigned to each vertex of the mesh allowing it to move in a force field governed by internal and external forces. In order to maintain regularity during deformation, each vertex is displaced by a small step $k, \mathbf{x}_{\mathbf{i}}=\left(\mathbf{x}_{\mathbf{i}-\mathbf{1}}+k \mathbf{n}\right)$, conditionally to the force field at $\mathbf{x}_{\mathbf{i}},\left\{F_{\text {ext }}\left(\mathbf{x}_{\mathbf{i}}\right) \geq F_{\text {int }}\left(\mathbf{x}_{\mathbf{i}}\right)\right\}$, where $\mathbf{n}$ denotes the mean normal vector in the neighborhood of $\mathbf{x}_{\mathbf{i}}$ (Fig. 2(b)].

External Forces. To perform the shape recovery, we introduce two external forces at each vertex, $F_{\text {ext }}=F_{I}+F_{\nabla}$. They represent the influence of the image on the embedded surface. The force $F_{I}$ guides the surface toward the image high intensity values while the force $F_{\nabla}$ drives the surface to regions of strong gradient. Both forces are normalized by the invariant maximum grey-scale value $\hat{I}$. External forces are computed from the raw data by transforming the discrete volumetric image $I$ into a continuous scalar field using tri-linear interpolation. The attraction toward an intensity value in this field is expressed by

$$
F_{I}\left(\mathbf{x}_{\mathbf{i}}\right)=\frac{I_{H E}\left(\mathbf{x}_{\mathbf{i}}\right)}{\hat{I}},
$$

where $I_{H E}$ denotes the histogram-equalized image $I$. The force $F_{I}$ aims to inflate the model locally at high intensity regions.

As grey-level values decrease when approaching the outer surface of the bronchial wall, $F_{\text {ext }}$ is strengthened by a force which drives the surface along the local gradient of the image:

$$
F_{\nabla}\left(\mathbf{x}_{\mathbf{i}}\right)=\frac{\left|\nabla_{i n}\left(\mathbf{x}_{\mathbf{i}}, I\right)\right|}{\hat{I}},
$$

where $I$ denotes the original image intensity and $\nabla_{i n}$ the lateral gradient computed with respect to the inner local neighborhood of $\mathbf{x}_{\mathbf{i}}, \mathcal{N}\left(\mathbf{x}_{\mathbf{i}-\mathbf{1}}\right)$, Fig. 2(b). The model is attracted to strong contours of the wall guided by the force $F_{\nabla}$, thus allowing to match wall irregularities.

Internal Forces. In order to equilibrate the external force, $F_{\text {int }}$ will be defined as a composition of two forces, $F_{i n t}=F_{\delta}+F_{r}$, an elastic force $F_{\delta}$, which linearly penalizes local wall thickness variations, and a regularization force $F_{r}$, which locally smoothes the shape. The elastic force is defined as the distance from the vertex $\mathbf{x}_{\mathbf{i}}$ to the inner wall surface mesh, $\delta\left(\mathbf{x}_{\mathbf{i}}, M_{0}\right)$, weighted by the mean distance computed over the vertices situated in a cross-section slab including $\mathbf{x}_{\mathbf{i}}$, $S_{\perp}\left(\mathbf{x}_{\mathbf{i}}\right)$, and orthogonal to the bronchus axis (Fig. 2(c)]:

$$
F_{\delta}\left(\mathbf{x}_{\mathbf{i}}\right)=\frac{\delta\left(\mathbf{x}_{\mathbf{i}}, M_{0}\right)}{\left(\mu_{\text {axial }}+\sigma_{\text {axial }}\right)}, \quad \mu_{\text {axial }}=\sum_{\mathbf{x}_{\mathbf{j}} \in S_{\perp}\left(\mathbf{x}_{\mathbf{i}}\right)} \frac{1}{\left|S_{\perp}\left(\mathbf{x}_{\mathbf{i}}\right)\right|} \delta\left(\mathbf{x}_{\mathbf{j}}, M_{0}\right),
$$

with $\mu_{\text {axial }}$ denotes the mean distance value to $M_{0}$ and $\sigma_{\text {axial }}$ the corresponding standard deviation. The shape of the external surface of the bronchus at the level of vessel-bronchus contact zone is mainly constrained by the elastic component to follow the inner wall contour shape. 
The regularization term, $F_{r}$, exploits the local normal curvature, computed according to the differential geometry definition [14] $: F_{r}=(-\nabla(A) / 2 A) \odot \mathbf{n}$, where $A$ denotes the local area around $\mathbf{x}_{\mathbf{i}}$ and $\odot$ the inner product. $\nabla(A)$ can be discretized in case of triangulated surfaces by the formula described in [14, leading to

$$
F_{r}\left(\mathbf{x}_{\mathbf{i}}\right)=-\frac{1}{4 A}\left(\sum_{j \in \operatorname{star}(i)}\left(\cot \alpha_{i j}+\cot \beta_{i j}\right)\left(\mathbf{x}_{\mathbf{i}}-\mathbf{x}_{\mathbf{j}}\right) \odot \mathbf{n}\right)
$$

where $\operatorname{star}(i)$ denotes the subset of neighbor vertices of $\mathbf{x}_{\mathbf{i}}$ and $\alpha_{i j}, \beta_{i j}$ the two angles opposite to the edge $\mathbf{x}_{\mathbf{i}}-\mathbf{x}_{\mathbf{j}}$ as illustrated in Fig. 2(d).

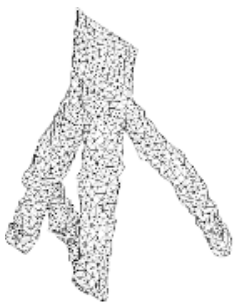

(a)

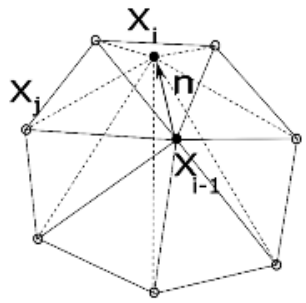

(b)

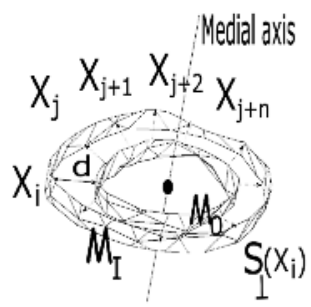

(c)

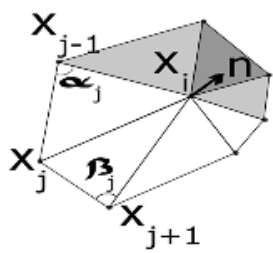

(d)

Fig. 2. Illustration of internal constraints associated with the deformable model: (a) Inner wall mesh, $M_{0}$, (b) Vertex motion, where $\left\{\mathbf{x}_{\mathbf{j}}\right\}_{j}=\mathcal{N}\left(\mathbf{x}_{\mathbf{i}-\mathbf{1}}\right)$, (c) Elastic force definition, (d) Regularization term definition

Mesh Resolution and Topology Adaptation. As a result of vertex displacement, the inter-vertices distance will change, leading either to skip important image features in case of too large distance, or to increase computational cost when too dense vertex distributions occur. In order to maintain adequate surface mesh resolution and high computational efficiency, the edge length is constrained during the deformation process, according to $\xi \leq d_{E}(u, v) \leq \lambda \xi$, where $d_{E}(u, v)$ denotes the Euclidean distance between two $u, v$ vertices, $\xi$ determines the global resolution of the surface, and $\lambda$ the length ratio between the longest and the shortest edges allowed. Edges not holding these constraints are removed or subdivided by methods used in progressive meshing [15]. The vertex displacement step between two iterations, $k$, as well as mesh density $\xi$, are set according to the highest CT data spatial resolution. They are iteratively decreased during the deformation process as approaching the solution, in order to reach the optimal mesh resolution at the convergence. Mesh deformation may also lead to surface self-intersections, especially in the case of bronchus subdivision. To prevent this problem, auto-collision is checked for each vertex 16. 


\section{Results and Discussion}

The bronchial quantification was cross-validated compared to the $2 \mathrm{D} / 3 \mathrm{D}$ reference method, with respect to a 3D image model simulating a cylindrical bronchus subdivision geometry for different calibers and lumen/wall textures [6] (Fig. 3] top). Only regions providing reliable quantification (outside the subdivision zone) were taken into account when applying the $2 \mathrm{D} / 3 \mathrm{D}$ method (Fig. 3(a)), whereas the quantification was applied to the whole model using the 3D method (Fig. 3(b) . The same cross-section planes have been used for both $3 \mathrm{D}$ and 2D/3D methods, for a quantification evaluation purpose. The percent error between the quantification provided by the developed approach and the model ground truth $(1.12 \% \pm 1.18 \%$ for lumen area, $1.18 \% \pm 1.72 \%$ for wall area) was not meaningfully different from that obtained with the $2 \mathrm{D} / 3 \mathrm{D}$ method $(0.27 \% \pm 0.57 \%$ for lumen area, $0.24 \% \pm 0.58 \%$ for wall area) and was always under $3 \%$. Comparisons carried out on real data for different distal bronchi at sampling points providing good discrimination of bronchus wall in the cross-section plane, showed similar results for the volumetric and the 2D/3D method (relative percent error: $1.08 \% \pm 1.57 \%$ for lumen area, $1.11 \% \pm 1.64 \%$ for wall area - Fig. 3 , bottom). These results tend to prove that the volumetric method has similar performance as the reference 2D/3D method for ideal situations and simple bronchus configurations. However, the volumetric approach is expected to provide higher accuracy and robustness

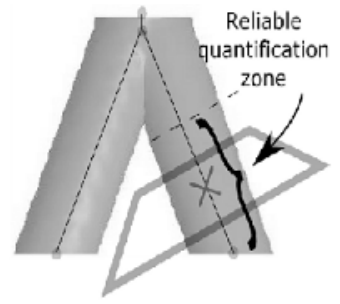

(a) Surface quantification
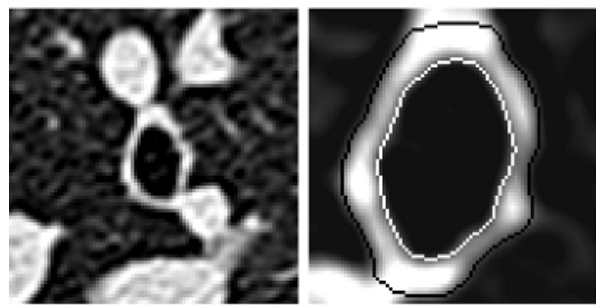

(c) Axial native image cation result
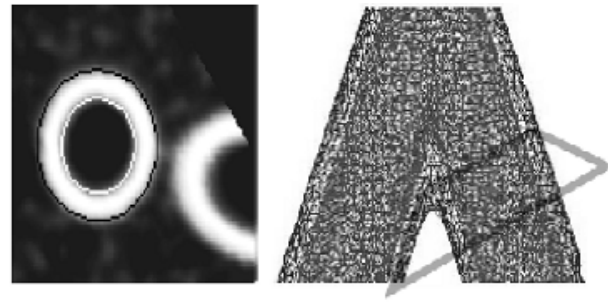

(b) Volumetric quantification

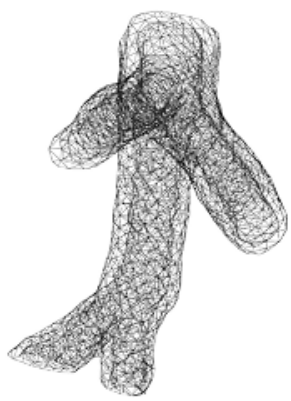

(d) Surface quantifi- (e) 3D segmentation

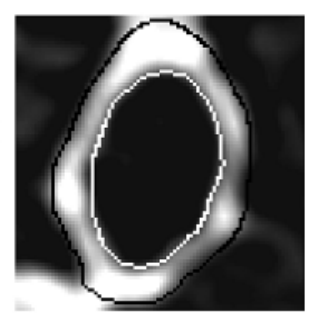

(f) Volumetric quantification result

Fig. 3. Surface (a)(c)(d) vs. volumetric (b)(e)(f) quantification of the bronchial wall on bronchus image phantom (top) and real data (bottom). 


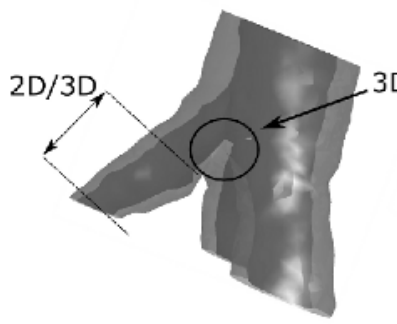

Before treatment

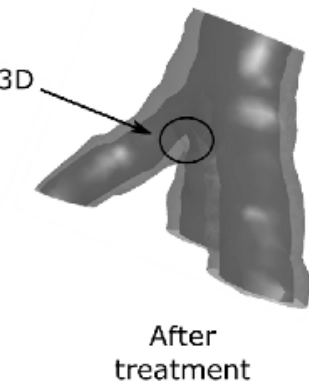

(a)

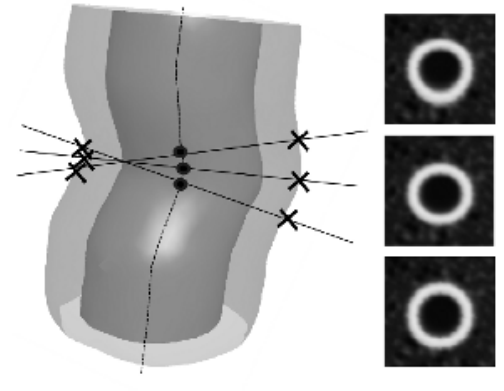

(b)

Fig. 4. Limitations of surface quantification approaches: (a) Restriction of the measurement zones, (b) Local deformation of the bronchus axis inducing estimation errors

in clinical situations by overcoming several limitations which are inherent to surface quantification methods, namely:

(1) a strong dependency on the correct estimation of the medial axis: deviations from the real tangent may cause wall area quantification errors.

(2) choice of sampling location in the bronchus: wall thickness is generally irregular along the bronchial segment. The solution retained in the $2 \mathrm{D} / 3 \mathrm{D}$ method is to provide an average value over each bronchus.

(3) measurement area dependency: only regions of the bronchus outside a subdivision area can be considered with the $2 \mathrm{D} / 3 \mathrm{D}$ method while the volumetric approach can capture wall thickness variations in this zones (Fig. 4(a))

(4) local bronchus irregularity may cause large local variations in the sampling plane orientation resulting in asymmetric and tilted sampling of wall regions (Fig. 4(b)].

(5) dependency of lung inflation during CT acquisition: due to their elastic properties, bronchi elongate at deep inspiration, which results in bronchus wall thinning. In such case, surface quantification will underestimate wall area while volumetric assessment will not be affected.

\section{Concluding Remarks}

Volumetric quantification of bronchial wall is recommended versus surface quantification for assessing small changes in airway wall remodeling in severe and mild asthmatics, before/after a therapeutic protocol. Due to the diffuse nature of asthma, such follow-up studies have to investigate a large number of distal bronchi spread throughout the lungs. Another advantage of the volumetric approach is that it can be applied to several bronchus generations, thus leading to more accurate measurements than any surface approach (cf. $\S$ 4). The developed volumetric method provides the investigation tools for designing new therapeutic protocols and assessing their efficiency with respect to various airway diseases. This technique is currently subject to a large-scale evaluation in the framework of a therapy follow-up study, jointly performed using the reference 2D/3D technique. 


\section{References}

1. S. Matsuoka, Y. Kurihara, Y. Nakajima, H. Niimi, H. Ashida, K. Kaneoya, "Serial Change in Airway Lumen and Wall Thickness at Thin-Section CT in Asymptomatic Subjects", in Radiology, 234 (2), pp. 595-603, 2005.

2. F. Chabat, X.P. Hu, D.M. Hansell, G.Z. Yang, "ERS transform for the automated detection of bronchial abnormalities on CT of the lungs", in IEEE transactions on medical imaging, 20, 2001.

3. G.G. King, N.L. Muller, K.P. Whittall, Q.S. Xiang, P.D. Pare, "An Analysis Algorithm for Measuring Airway Lumen and Wall Areas from High-Resolution Computed Tomographic Data", in Am. J. R. Cr. Care Med., 161, pp. 574-580, 2000.

4. R. Wiemker, T. Blaffert, T. Blow, S. Renisch, C. Lorenz, "Automated assessment of bronchial lumen, wall thickness and bronchial tree using high-resolution CT", in nternational Congress Series, 1268, pp. 967-972, 2004.

5. E.A. Hoffman, J.M. Reinhardt, M.Sonka, et al., "Characterization of the intersistal lung diseases via density based and texture-based analysis of computed tomography images of lung structure and function", in Acad. Radiology, 10, pp. 1104-1118, 2003 .

6. A.Saragaglia, C.Fetita, F.Prêteux, PY Brillet, P. Grenier, "Accurate 3D quantification of bronchial parameters in MDCT", Proceedings SPIE Conference on Mathematical Methods in Pattern and Image Analysis, 5916, pp. 323-334, 2005.

7. C. Fetita, F. Prêteux, C. Beigelman-Aubry, P. Grenier, "Pulmonary airways: 3D reconstruction from multi-slice CT and clinical investigation", IEEE Transactions on Medical Imaging, 23(11), pp. 1353-1364, 2004.

8. D. Perchet, C. Fetita, F. Prêteux, "Physiopathology of Pulmonary Airways: Automated Facilities for Accurate Assessment", MICCAI, 2, pp. 234-242, 2004.

9. PY. Brillet , C. Fetita, C. Beigelman-Aubry, D. Perchet, F. Prêteux,P. Grenier, "Automatic segmentation of airway wall area for quantitative assessment at MDCT: preliminary results in asthmatics", in European Congress of Radiology, 2005.

10. P. Brillet, A. Saragaglia, C. Fetita, C. Beigelman-Aubry, S. Dreuil, F. Prêteux, P. Grenier, "Automatic quantification of bronchi using MDCT: Comparison of FWHM and EDCE methods on computerized simulations and in vivo bronchi", in European Congress of Radiology, 2006.

11. J.-D. Boissonnat and S. Oudot "Provably Good Surface Sampling and Approximation", in Eurographics Symposium on Geometry Processing, 2003.

12. L.P. Chew, "Guaranteed-Quality Mesh generation for Curved Surfaces", in Proc. 9th Annu. ACM Sympos. Comput. Geom. , 10, pp. 274-280, 1993.

13. D. Perchet, "Modelisation in-silico des voies aeriennes : reconstruction morphologique et simulation fonctionnelle", PHD Thesis, Univ. Paris 5, November 2005.

14. M. Desbrun, M. Meyer, and P. Alliez, "Intrinsic Parameterizations of Surface Meshes", in Computer Graphics Forum, 21(3), pp. 209-218, 2002.

15. H. Hoppe, T. DeRose, T. Duchamp, J. McDonald and W. Stuetzle, "Mesh optimization", in Computer Graphics (SIGGRAPH 93 Proceedings), pp. 1926, 1993.

16. J.-O. Lachaud and B. Taton, "Deformable model with adaptive mesh and automated topology changes", in Proc. 4th Int. Conference on 3D Digital Imaging and Modeling, pp. 1219, IEEE, 2003. 